\title{
Etude hydrométéorologique du Bassin de Sarennes Présentation du projet et premiers résultats
}

\author{
Par E. MARTIN, Y LEJEUNE, Météo-France/CNRM/CEN \\ E. LEBLOIS, Cemagref-HH Lyon \\ M. COLLOMBET, F. VALLA, D. BIRONNEAU, Cemagref-nivologie Grenoble \\ E. LEDOUX, C. CAVAZZI Ecole des Mines de Paris/CIG Fontainebleau
}

\section{I : CONTEXTE ET PRÉSENTATION DU PROJET}

Le manteau neigeux joue un rôle très important dans le régime des rivières des régions montagneuses tempérées. C'est évidemment le cas des rivières des Alpes françaises, dont les bassins supérieurs sont à des altitudes suffisamment élevées pour supporter un manteau neigeux significatif durant l'hiver. Les effets du manteau neigeux sur les apports d'eau sont multiples et contradictoires :

- Le manteau neigeux constitue une réserve très importante d'eau libérée en quelques semaines ou quelques mois lors de la période de fonte, avec des lames d'eau quotidiennes de 15 à $30 \mathrm{~mm}$. Ces quantités importantes d'eau peuvent saturer les sols, rendant ceux-ci plus sensibles à un épisode de précipitations marqué.

- Le manteau neigeux peut aussi dans certains cas limiter les risques de crue lors d'épisode de fortes précipitations lorsque la limite pluie/neige est suffisamment basse pour que les précipitations soient stockées sous forme de neige à haute altitude. Dans le cas de chutes de pluie sur un manteau sec, une partie des précipitations peut être retenue dans le manteau neigeux.

Le but du projet est de quantifier les phénomènes décrits ci-dessus sur le bassin nivo-glaciaire de Sarennes. En effet, ce bassin est suivi depuis de nombreuses années par le Cemagref pour sa composante glaciaire, et des chroniques de débit sont disponibles à plusieurs altitudes : $2735 \mathrm{~m}$, $2200 \mathrm{~m}$ (Cemagref), $1435 \mathrm{~m}$ (EDF). Cela permettra de séparer la part des débits liée à la pluie, la fonte de la neige saisonnière ou celle du glacier.

L'étude est basée sur la modélisation hydrométéorologique du bassin à l'aide du couplage d'un système d'analyse météorologique, d'un modèle d'évolution du manteau neigeux et d'un modèle hydrologique distribué.

Cette action de recherche (retenue dans le cadre du XIe contrat de plan Etat-Région Rhône-Alpes pour la Recherche) devrait durer deux ans environ. Elle est menée en collaboration entre le Centre d'études de la neige, la division $\mathrm{HH}$ du Cemagref/Lyon, la division nivologie du Cemagref/Grenoble et l'Ecole des Mines de Paris. Les principales étapes du projet sont :
- Mesures hivernales sur le glacier de Sarennes, dans le but de quantifier les précipitations locales, la redistribution de la neige par le vent (Cemagref, CEN)

- Analyse des conditions météorologiques, simulation du manteau neigeux par les outils SAFRAN et CROCUS sur la période 1981/1996. Validation avec les mesures effectuées sur le glacier (CEN/Cemagref).

- Adaptation du modèle hydrologique de l'Ecole des Mines au bassin de Sarennes (Cemagref/Ecole des Mines)

- Analyse de l'aléa de crue (Cemagref/Ecole des Mines/CEN)

\section{II 圈 MESURES SUR LE GLACIER (HIVER 95/96)}

Le bassin versant amont de Sarennes (exutoire $2735 \mathrm{~m}$ ) représente une superficie globale de 123 ha, dont 52 ha recouverts par le glacier. La campagne de mesures entamée début janvier 1996 consiste à suivre l'évolution des hauteurs de neige en plusieurs points situés dans des zones variées du point de vue de l'exposition, pente et topographie locale.

Des mesures hebdomadaires sont réalisées sur un réseau de 26 perches à neige. Celles-ci sont disposées selon deux profils principaux, alignés sur un axe nord/sud. Le profil gauche débute à $3095 \mathrm{~m}$ sur un col situé à $200 \mathrm{~m}$ à l'ouest du chalet du GUC et se termine à $2930 \mathrm{~m}$ (col de Chateau Noir, figure 1). Il comprend deux cols, sites privilégiés pour l'observation du transport de neige par le vent, ainsi qu'une zone de dépôts d'avalanches. Le second profil s'étend de $3250 \mathrm{~m}$ à $2840 \mathrm{~m}$ sur la rive droite du glacier, à partir des pentes sommitales du Pic Blanc.

L'analyse des données est en cours, mais il est d'ores et déjà possible de classer les perches en trois familles dont la répartition est donnée dans le tableau 1.

La figure 2 permet de comparer l'évolution de la hauteur de neige au niveau de différentes perches situées sur le bas$\sin$. On a également reporté la hauteur de neige au poste nivométéorologique de l'Alpe d'Huez (2 $300 \mathrm{~m})$. La différence entre la hauteur de neige au col de Château Noir (particulièrement venté) et la perche D13, située à la limite inférieure du glacier (où l'accumulation est très forte) est très importante. 


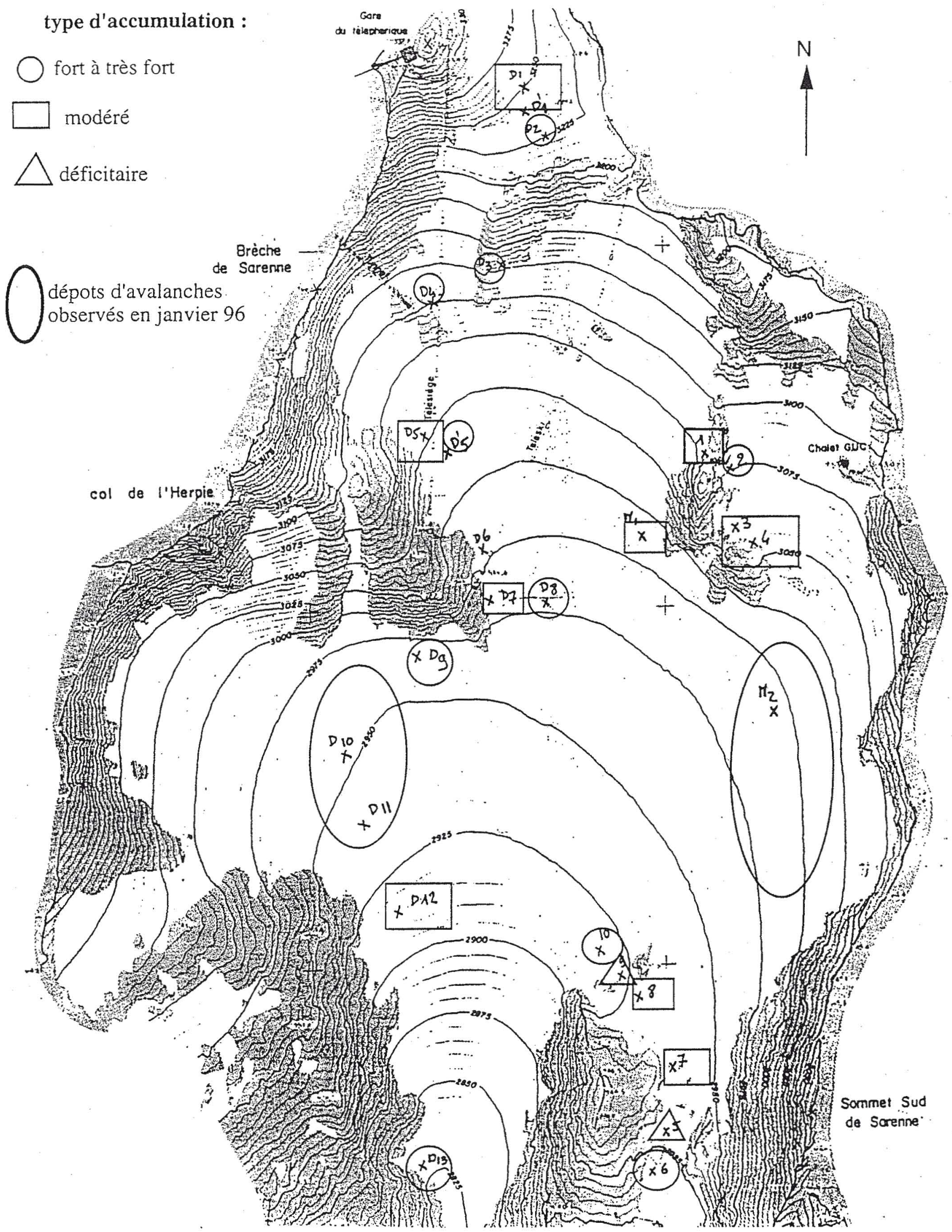

1. Carte du glacier de Sarennes avec indication de l'emplacement des perches. 


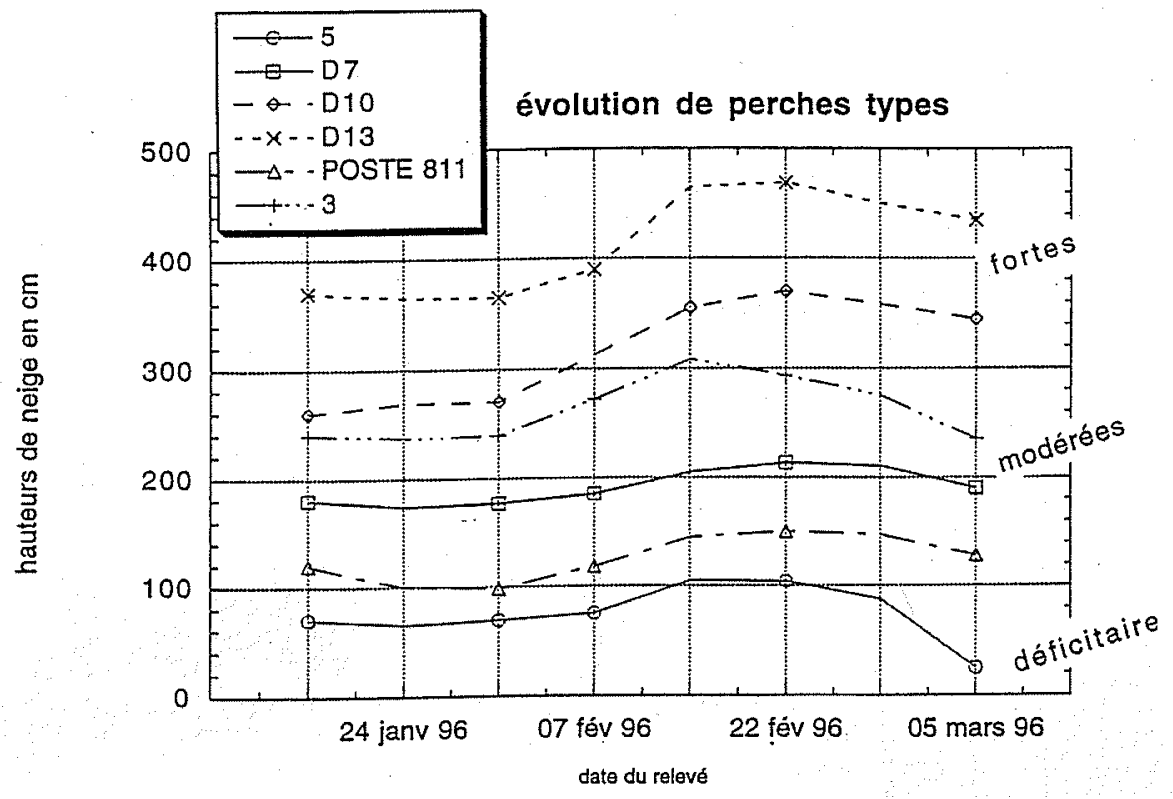

2. Comparaison des hauteurs de neige au niveau de différentes perches. Le poste nivométéorologique 811 est situé à une altitude de $2300 \mathrm{~m}$ sur le domaine de l'Alpe d'Huez.

GLACIER DE SARENNE Periode 1981/1995

Comparaison des bilans (mesures/simulations) Flchler :bllan.gmt-c

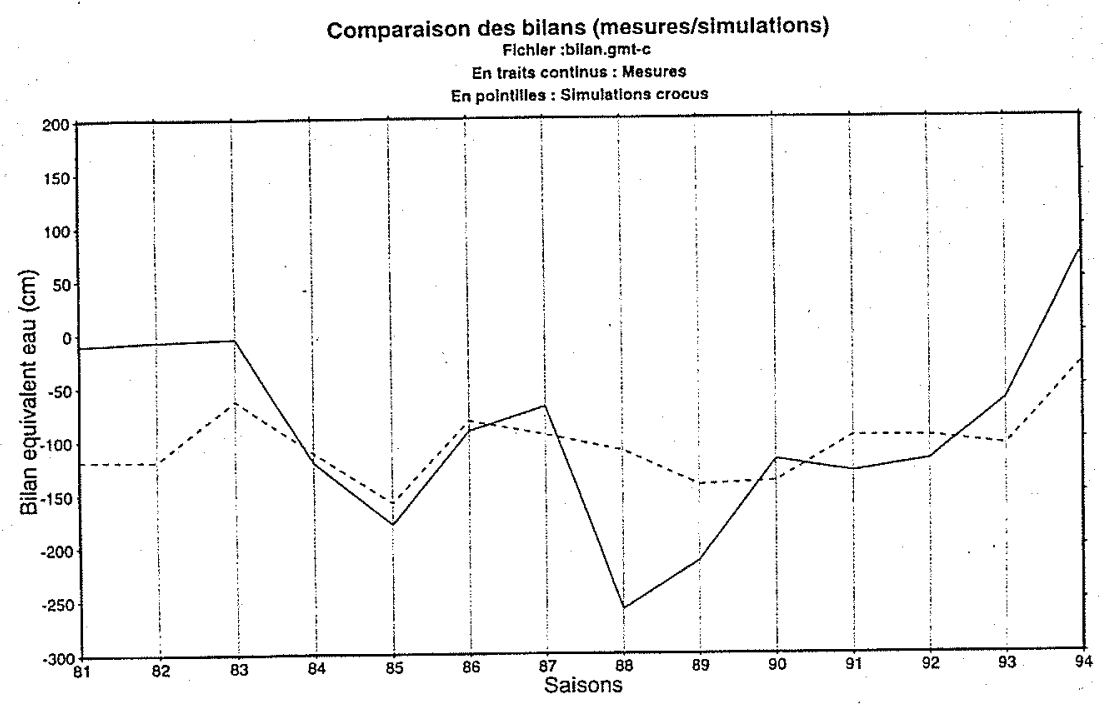

3. Comparaison des bilans de masse simulés et mesurés.

En haut : simulation 1, en bas, simulation 2 . Chaque hiver est repéré par l'année de début : $81=$ hiver $81 / 82$.

GLACIER DE SARENNE Periode 1981/1995

Comparaison des accumulations (mesures/simulations) Fichier :sccu.gmt-e

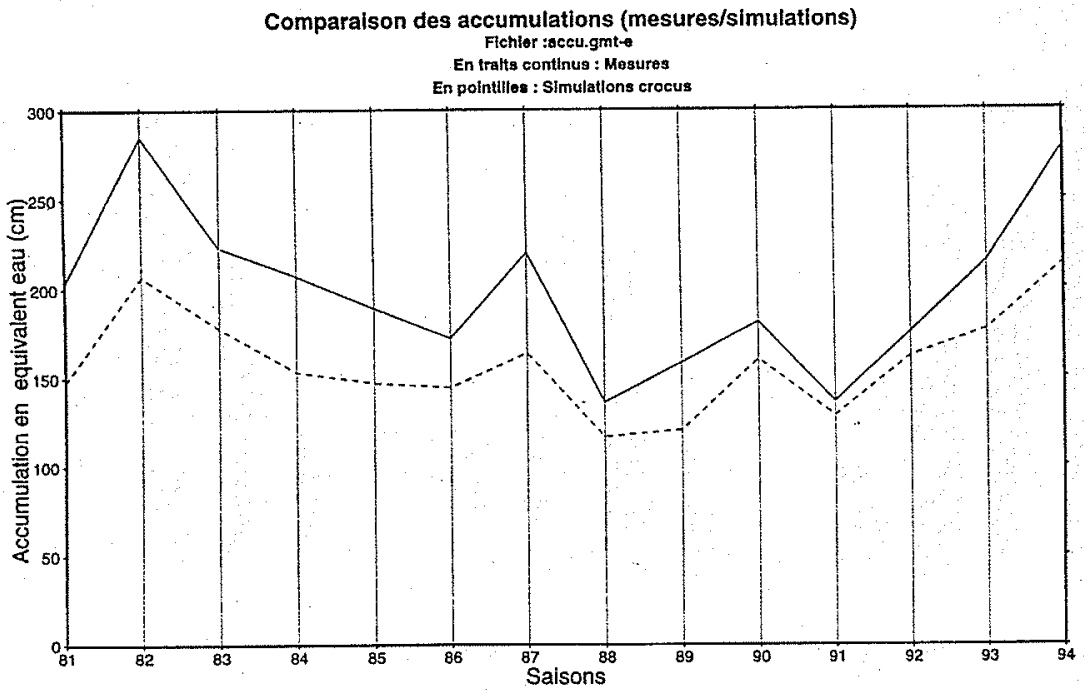

4. Comparaison des accumulations simulées et mesurées.

En haut : simulation 1, en bas, simulation 2 .

Chaque hiver est repéré par l'année de début : $81=$ hiver $81 / 82$. 


\begin{tabular}{|c|c|c|}
\hline type de comportement & nom des perches & $\begin{array}{c}\text { représentativité par rapport } \\
\text { à la totalité des perches }\end{array}$ \\
\hline $\begin{array}{c}\text { très fortes et fortes } \\
\text { accumulations }\end{array}$ & $\begin{array}{c}\text { M2, D9, D11, D13 } \\
2,6, \mathrm{D} 2, \mathrm{D} 3, \mathrm{D} 4, \mathrm{D} \text { '5, } \\
\mathrm{D} 8, \mathrm{D} 10\end{array}$ & $50 \%$ \\
\hline $\begin{array}{c}\text { accumulations assez fortes } \\
\begin{array}{c}3,4,8, \mathrm{D} 1, \mathrm{D} \text { '1, D5, D12 } \\
1,7, \mathrm{M} 1, \mathrm{D} 7\end{array}\end{array}$ & $42 \%$ \\
\hline $\begin{array}{c}\text { pas ou très peu } \\
\text { d'accumulation }\end{array}$ & 5,9 & $8 \%$ \\
\hline
\end{tabular}

Tableau 1. - Classification des perches à neige selon leur comportement.

\section{PREMIERES SIMULATIONS DU BILAN DE MASSE DU GLACIER}

Parallèlement aux mesures, des essais de modélisation du bilan de masse du glacier ont été effectués grâce au système d'analyse SAFRAN et au modèle CROCUS utilisés depuis quelques années pour la simulation en temps réel du manteau neigeux au CEN. Les données météorologiques d'entrée de SAFRAN (sorties de modèles météorologiques, observations) depuis le ler août 1981 sont disponibles au CEN. Elles ont été cependant complétées par les observations des postes climatologiques des Alpes, non transmises automatiquement. Ces données peuvent être très importantes car elles prolongent ou complètent pendant l'automne et le printemps les observations des postes nivométéorologiques et augmentent la qualité des analyses météorologiques (en particulier, les postes suivants ont été ajoutés : Allemont, Auris, Besse, Huez, Ornon, Vaujany). SAFRAN a été activé avec ces données sur la région des Grandes Rousses du ler août 1981 au 31 janvier 1996. Ainsi, on dispose de paramètres météorologiques au pas horaire de 1200 à $3300 \mathrm{~m}$ par pas de $300 \mathrm{~m}$ sur plusieurs orientations.

Les simulations du bilan du glacier ont été réalisées avec le modèle CROCUS, dont le but initial est la modélisation de la neige saisonnière. Certaines adaptations pour les simulations glaciaires ont dû être faites :

- Introduction de couches de glace à la base du manteau neigeux (prises comme étant de la neige à $917 \mathrm{~kg} \cdot \mathrm{m}^{-3}$. Certaines lois physiques (conduction, tassement) ont été adaptées en conséquence.

- Introduction d'un albédo fixe lorsque les couches de glace sont en surface.
- Diminution du flux thermique du sol. Les valeurs habituelles de l'ordre de quelques $\mathrm{Wm}^{-2}$ sont justifiées lorsque le sol peut emmagasiner de la chaleur pendant l'été, mais ce n'est évidemment pas le cas du glacier de Sarennes. Le flux habituellement utilisé a été divisé par 100 , ce qui correspond à une fonte à la base négligeable (de l'ordre de quelques mm d'eau sur les 14 années).

Plusieurs simulations correspondant à un point situé à $3000 \mathrm{~m}$ d'altitude au centre du glacier ont été effectuées pour évaluer la sensibilité des simulations à divers paramètres. Dans tous les cas, le profil initial le premier août 1981 est constitué de $20 \mathrm{~m}$ de glace (18.34 m d'équivalent en eau). On décrira ici les résultats de deux simulations, dont les principales caractéristiques sont reprises dans le tableau 2.

La simulation 1 est très proche des simulations effectuées dans le cadre de la chaine SAFRAN/CROCUS/MEPRA et servira de comparaison pour les simulations ultérieures. La simulation 2 correspond à une situation plus proche de la réalité : orientation du glacier au sud avec pente de $15^{\circ}$, augmentation des quantités de précipitations car l'analyse SAFRAN est trop faible sur cette zone.

Pour l'analyse, des données simulées d'équivalent en eau de la glace et de la neige au pas quotidien ont été utilisées. Ces données ont été comparées aux mesures effectuées par le Cemagref depuis de nombreuses années : accumulation, ablation, bilan (accumulation - ablation) et régime (accumulation + ablation). Sur la période considérée (cycles 34 à 47 soit 14 cycles, tableau 3 ), le bilan de masse du glacier est négatif $(-13,1 \mathrm{~m})$. Lorsque l'on s'intéresse aux simulations, on constate que le bilan est de $-14,7 \mathrm{~m}$ pour la simulation 1 et de $-8,2 \mathrm{~m}$ pour la simulation 2 .

\begin{tabular}{|c|c|c|c|c|c|}
\hline $\begin{array}{c}\text { numéro de } \\
\text { la simulation } \\
\cdot\end{array}$ & altitude $(\mathrm{m})$ & $\begin{array}{c}\text { Hauteur } \\
\text { glace initiale } \\
(\mathrm{m})\end{array}$ & $\begin{array}{c}\text { Exposition } \\
\text { et pente }\end{array}$ & $\begin{array}{c}\text { albédo } \\
\text { de la glace } \\
\text { (ciel clair) }\end{array}$ & $\begin{array}{c}\text { Coefficient } \\
\text { multiplicatif } \\
\text { pour les } \\
\text { précipitations }\end{array}$ \\
\hline 1 & 3000 & 20 & plat & 0.37 & 1. \\
\hline 2 & 3000 & 20 & Sud $/ 15^{\circ}$ & 0.34 & 1.5 \\
\hline
\end{tabular}

Tableau 2. - Principales caractéristiques des simulations CROCUS 


\begin{tabular}{|l|c|c|c|}
\hline paramètre & $\begin{array}{c}\text { Mesures } \\
\text { équivalent en eau (m) }\end{array}$ & $\begin{array}{c}\text { simulation 1 } \\
\text { équivalent en eau (m) }\end{array}$ & $\begin{array}{c}\text { simulation 2 } \\
\text { équivalent en eau (m) }\end{array}$ \\
\hline bilan cumulé & $-13,1$ & $-14,7$ & $-8,2$ \\
\hline acumulation totale & 27,8 & 15,2 & 22,1 \\
\hline ablation totale & 40,9 & 30,0 & 30,3 \\
\hline $\begin{array}{l}\text { régime annuel } \\
\text { moyen }\end{array}$ & 4,9 & 3,2 & 3,7 \\
\hline
\end{tabular}

Tableau 3. - Comparaison des mesures sur les 14 cycles aux simulations.

Même si le bilan cumulé de la première simulation est plus proche des mesures dans la simulation 1, l'examen des bilans annuels (figure 3 ) montre que les bilans positifs ou nuls sont nettement sous évalués $(81 / 82,82 / 83,83 / 84$ et 94/95). La variabilité interannuelle est globalement respectée à l'exception notable des années $88 / 89$ et $89 / 90$, périodes de fortes ablations non reproduites dans les simulations. La variabilité interannuelle de l'accumulation est correctement reproduite, malgré une sous-estimation systématique (figure 4). Les erreurs importantes sur le bilan en $88 / 89$ et $89 / 90$ sont donc imputables à la phase d'ablation.

Dans le cas de la simulation 2, l'accumulation est plus proche des mesures, quoique toujours sous-estimée. Pour ce qui concerne l'ablation, deux effets se compensent à peu près :

- l'augmentation de l'accumulation entraine un allongement de la durée pendant laquelle la neige recouvre le glacier (fonte plus faible)
- l'introduction d'une exposition et d'une pente orientée au sud a tendance à accélérer la fonte.

L'ablation reste donc a un niveau comparable à celle de la simulation 1 (toujours insuffisante). Le bilan cumulé simulé est donc plus mauvais que celui de la simulation 1 .

\section{CONCLUSION}

Les mesures en cours sur le glacier de Sarennes permettront de mieux connaitre la variabilité spatiale de l'enneigement sur ce site. Il semble en effet qu'il y ait des différences importantes entre les hauteurs mesurées an niveau des postes nivométéorologiques (1850 et $2300 \mathrm{~m}$ ), les données de SAFRAN et CROCUS et l'accumulation constatée sur les différentes perches. Pour ce qui concerne la partie modélisation, les résultats obtenus sont du même ordre de grandeur que les mesures, par contre le modèle de neige CROCUS sera amélioré pour mieux simuler la phase d'ablation de la glace, qui est pour l'instant la partie la plus mal simulée. 